\title{
PROBability OF Violation OF The Ehrenfest PRinciple IN FAST PASSAGE
}

\author{
GREGORY H. WANNIER * \\ Bell Telephone Laboratories, Inc. \\ Murray Hill, New Jersey
}

ADIABATIC passage through an almost degenerate intermediate state is a common experimental techrique. It is based on the Ehrenfest principle which states that if the external conditions for a system are changed slowly enough, the change of the system can be predicted by computing quasistatically the modification of its initial quantum state. The Ehrenfest principle is not a law of nature because the system has an alternate path it can follow, which might appropriately be termed 'inertial'. This path is favored by a rapid change of the external variables. Among the two paths, the adiabatic one has a privileged position. Thus, it has been shown recently that if the external change is measured by a "slowness parameter" inversely proportional to the time taken to effect the change then the Ehrenfest principle is valid to all orders in this parameter [1]. The following lines are to derive a rigorous formula for the probability that the adiabatic path is not followed in the simple case of the near crossing of two quantum levels.

The general problem of two levels crossing in time contains several parameters some of which are irrelevant. After they are disposed of we are left with the two essential parameters of the problem, namely, the sweeping rate $r$ at which energy difference of the two levels changes when we are not near to the crossing, and the minimum value $s$ which this difference takes at the time of closest approach. These parameters are shown in Fig. 1 in an energy time plot. The adiabatic paths of the systems are the two branches of the hyperbola, and the inertial paths are the asymptotes. If we treat the Hamiltonian as diagonal with respect to these inertial paths, the adiabatic tendency appears as an off-diagonal splitting factor, thus:

$$
\mathscr{H}=\left[\begin{array}{ccc}
1 / 2 r t & 1 / 2 s \\
1 / 2 s & -1 / 2 r & t
\end{array}\right]
$$

where $r$ and $s$ have the meanings discussed above. We now have to solve the schrơdinger equation

or

$$
i \not h \frac{d}{d t}\left(\begin{array}{l}
a \\
b
\end{array}\right)=\mathscr{H}\left(\begin{array}{l}
a \\
b
\end{array}\right)
$$

$$
\begin{aligned}
& i \not h \frac{d s}{d t}=1 / 2 r t a+1 / 2 s b \\
& i \not h \frac{d b}{d t}=1 / 2 s a-1 / 2 r t b
\end{aligned}
$$

* Present address: University of Oregon, Eugene, Oregon 


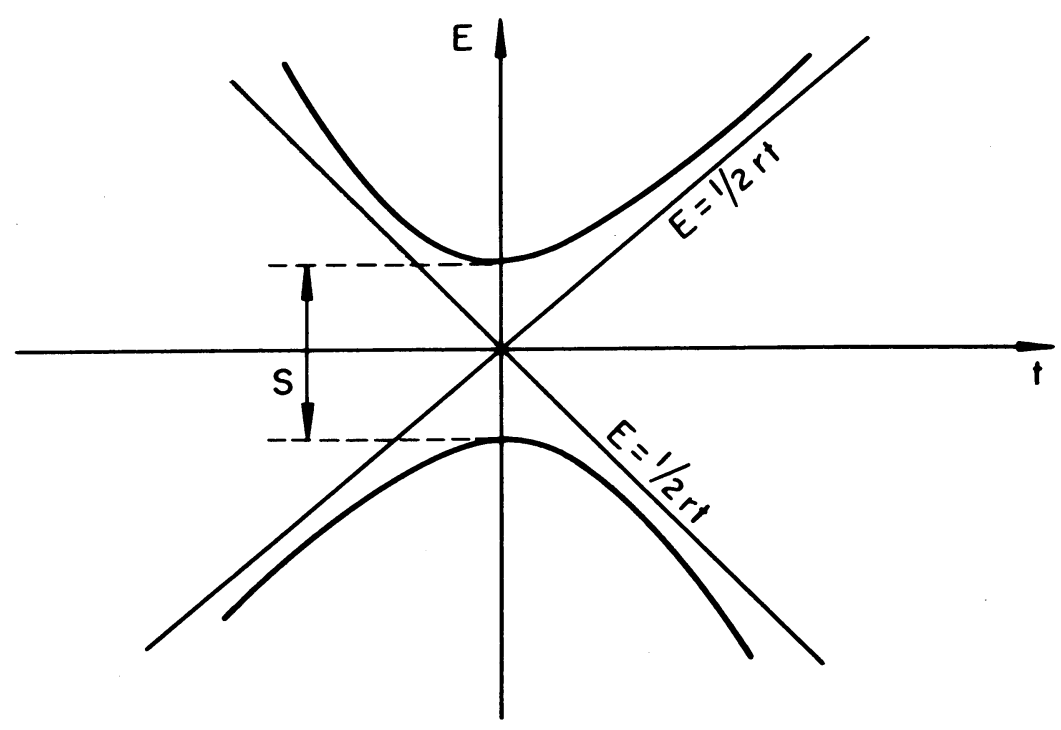

FIGURE 1

Elimination of $b$ from this system yields

$$
\frac{d^{2} a}{d t^{2}}+\left\{\frac{r^{2} t^{2}}{4 h^{2}}+\frac{s^{2}}{4 h^{2}}+\frac{i r}{2 h}\right\} a=0
$$

This is the equation for a parabolic cylinder function, a special type of confluent hypergeometric function. Its asymptotics is completely understood [2]. In terms of Whittaker functions equation (3) has the two solutions

$$
\begin{aligned}
& a_{1}(t)=t^{-Z_{2} W_{1}} \frac{i s^{2}}{8 r h,} \frac{1}{4}\left(\frac{i r t^{2}}{2 h}\right) \\
& a_{2}(t)=t-\frac{1}{8} W_{1}-\frac{i s^{2}}{4}+\frac{1}{8 r h,}\left(-\frac{i r t^{2}}{2 / h}\right)
\end{aligned}
$$

Let us now assume that positive $t$ points into the past and that in this remote past the system was with certainty in the state characterized by $a$. We must then write down the asymptotic expressions for the solutions (4) which are, with a modification of the factor

$$
\begin{aligned}
& a_{1}(t) \sim \exp \left[-\frac{i r t^{2}}{4 h}-\frac{i s^{2}}{4 r h} \ln t\right] \\
& a_{2}(t) \sim t^{-1} \exp \left[\frac{i r t^{2}}{4 h}+\frac{i s^{2}}{4 r h} \ln t\right]
\end{aligned}
$$

The solution ( $5 a)$ corresponds to a situation where the probability is finite that state $a$ was occupied in the remote past. In solution $(5 b)$ this same probability is zero. One can check from (2b) that the companion probabilities for the state $b$ are inverted, being finite for the second 
solution and zero for the first. If we assume that we start out with the system in state $a$, we must adopt the solutions $a_{1}(t)$ and its companion $b_{1}(t)$ and derive from analysis the value of $a_{1}(t)$ in the remote future, that is $t=-\infty$.

One may verify that the asymptotic expansion (5a) of the function (4a) is valid in the first, fourth and third quadrant of the complex plane, including the limiting lines. It is therefore also valid for negative $t$ provided we introduce the interpretation

$$
-t=e^{-i \pi} \mid t
$$

Inserting this into (5a) we get for the amplitude $a(t)$ in the remote future

$$
a(-t) \sim \exp \left[-\frac{i r t^{2}}{4 h}-\frac{i s^{2}}{4 r h} \ln |t|-\frac{\pi s^{2}}{4 r k}\right]
$$

The last term gives the reduction in amplitude along the inertial path (asymptote of Fig. 1). Its square measures therefore the probability that the Ehrenfest principle is violated. This probability $P$ is thus

$$
P=\exp \left[-\frac{\pi s^{2}}{2 r h}\right]
$$

Formula (8) is incidentally an example for the general theorems discussed in reference [1]. When the 'slowness parameter' $r$ is small, the probability $P$ is also small in such a way as to be smaller asymptotically than any finite power of $r$. The limiting case of fast passage, that is large $r$, may be derived by simpler methods [3].

\section{REFERENCES}

1. R.M. KULSRUd, Phys. Rev. 106, 205 (1957). Andrew Lenard, Report NY0-2393, Project Matterhorn, Princeton, December 1958.

2. E.T. WhITTAKER and G.N. MATsON, A Course of Modern Analysis, Chapter XVI.

3. In electrical engineering formula (8) may be relevant for the case of Cook's directional couplers. See B.S.T.J. 34, 807-870 (1955). 